\title{
Amplitude Modulation of EPSPs in Motoneurons in Response to a Frequency-Modulated Train in Single la Afferent Fibers
}

\author{
W. F. Collins, III, B. M. Davis, ${ }^{1}$ and L. M. Mendell \\ Department of Neurobiology and Behavior, SUNY at Stony Brook, Stony Brook, New York 11794
}

In anesthetized cats, single group Ia fibers were activated with a train of 52 stimuli whose interspike intervals were obtained from the discharge of a group Ia fiber in a walking cat (courtesy of Dr. G. E. Loeb, NIH). The EPSPs recorded in motoneurons to which the afferents projected were averaged in register $\left(\mathrm{EPSP}_{1}, \mathbf{E P S P}_{2}, \ldots, \mathrm{EPSP}_{52}\right)$ in response to multiple presentations of the train at a rate $(0.77 \mathrm{~Hz})$ similar to the stepping rate of the cat. Amplitudes of these averaged EPSPs were found to be highly variable, depending both on afferent discharge rate and history of activation. The initial EPSP was generally potentiated, and EPSPs in the high-frequency $(>200 \mathrm{~Hz}$ ) middle portion of the train were generally reduced in amplitude. Connections at which the first EPSP was most potentiated tended to be those which showed the most depression of EPSP amplitude during the high-frequency portion of the train. Thus, at some connections, modulation of EPSP amplitude during the frequency-modulated train was much greater than at other connections. In general, the extent of modulation was greater on low-rheobase motoneurons than on high-rheobase motoneurons. We suggest that these differences in transmission permit type $S$ (i.e., low-rheobase) motoneurons, which on average generate the largest EPSPs, to reach threshold at low levels of input (size principle) but prevent excessive depolarization due to temporal summation during high-frequency stimulation.

Numerous studies in sensory physiology have demonstrated that coding of stimulus parameters is accomplished in part by frequency modulation of the neuronal discharge (reviewed in Somjen, 1972). Similar principles appear to operate on the motor side of the nervous system at several different levels, e.g., the motoneuron (reviewed in Burke, 1981) and the motor cortex (reviewed in Evarts, 1981). A question that has received much less attention is the manner in which frequency-modulated discharges in presynaptic fibers are transmitted across the various synaptic relays intercalated in the neural pathways, in particular, the uniformity of such transmission.

This issue can be approached experimentally in a convenient way at the connection made by group Ia afferent axons supplying primary muscle spindle receptors on alpha-motoneurons. It is now well established that single group la fibers branch profusely to synapse on virtually all of the motoneurons of the homonymous motoneuron pool (Mendell and Henneman, 1971; see review in Henneman and Mendell, 1981). The motoneuron pool is heterogeneous, with some cells supplying slowly contracting

\footnotetext{
Received July 22, 1985; revised Oct. 24, 1985; accepted Oct. 30, 1985.

We thank Drs. J. B. Munson and T. C. Cope for reading an early draft of this manuscript. We are grateful to Dr. G. E. Loeb for providing the discharge of the spindle used in this work. W.F.C. was supported by NRSA NS-06407. B.M.D. was supported by NRSA NS-07319. The research was supported by NIH Grant NIH NS 16996, with some support from Program Project Grant NS 14899.

Correspondence should be addressed to Dr. Mendell at the above address.

'Present address: Department of Neurobiology, Northwestern University, Evanston, IL 60201 .

Copyright (c) 1986 Society for Neuroscience $0270-6474 / 86 / 051463-06 \$ 02.00 / 0$
}

motor units, type $S$, and others innervating fast contracting motor units, types FF and FR (see Burke, 1981, for a review). The motoneurons innervating these motor units can be distinguished with a relatively high degree of reliability by measuring their rheobase. Type $S$ motoneurons exhibit values of rheobase lower than $10 \mathrm{nA}$ and type $\mathrm{F}$ units have rheobase values typically greater than $10 \mathrm{nA}$, with some overlap (Fleshman et al., 1981a; Zengel et al., 1985). Thus, experimentally one can ask whether synapses made by la fibers on these diverse alpha-motoneurons have similar properties during stimulation at physiological frequencies.

In recent work from this laboratory single group Ia axons were stimulated by an intrafiber electrode, and the resultant EPSPs were recorded in homonymous alpha-motoneurons to which they projected (Collins et al., 1984; Davis et al., 1985; Honig et al., 1983). This technique permitted stimulation at the high frequencies $(>100 \mathrm{~Hz})$ that recordings from group Ia fibers in walking animals have revealed to be common (I oeb and Duysens, 1979; Prochazka et al., 1976, 1977). The principal finding of these experiments was that Ia fiber/alpha-motoneuron connections differed substantially in the way in which they transmit such high-frequency impulse trains. Connections generating small EPSPs tended to produce successively larger EPSPs up to some plateau value during high-frequency stimulation, a phenomenon we have termed "facilitation" (Collins et al., 1984b). Similarly, large EPSPs exhibited a tendency to diminish in amplitude during the same high-frequency stimulus trains, an effect referred to as "depression." Such connections are distributed in a nonrandom way to type $S$ and type $F$ motoneurons (Collins et al., 1984b; Fleshman et al., 1981a). Thus, these differences in response to high-frequency stimulation are distributed in a nonrandom manner in low- and high-rheobase motoneurons (see Fig. $6 B$ in Collins et al., 1984b). More recently, we have found that potentiation of transmission that follows such high-frequency stimulation differs in magnitude and time course in accordance with the amplitude of the EPSP (Davis et al., 1985). Such studies indicate that transmission from la fibers to the different motoneurons of a pool could be quite different both during and after high-frequency bursts of physiological frequency.

One problem with assessing the physiological relevance of such experiments is that single frequencies of stimulation were used. During locomotion, the discharge rate of single Ia fibers varies considerably, with the highest frequencies being achieved for only a few impulses. Thus, we have here adopted a different strategy: Instead of delivering bursts at constant frequencies (Collins et al., 1984b; Honig et al., 1983), we use frequencymodulated discharge patterns taken directly from hindlimb spindle discharges recorded from a walking cat. These results have been presented in abstract form (Collins et al., 1984a).

\section{Materials and Methods}

These experiments were carried out in 10 anesthetized cats (Nembutal $35 \mathrm{mg} / \mathrm{kg}+$ supplementary doses to maintain areflexia). The lumbar 


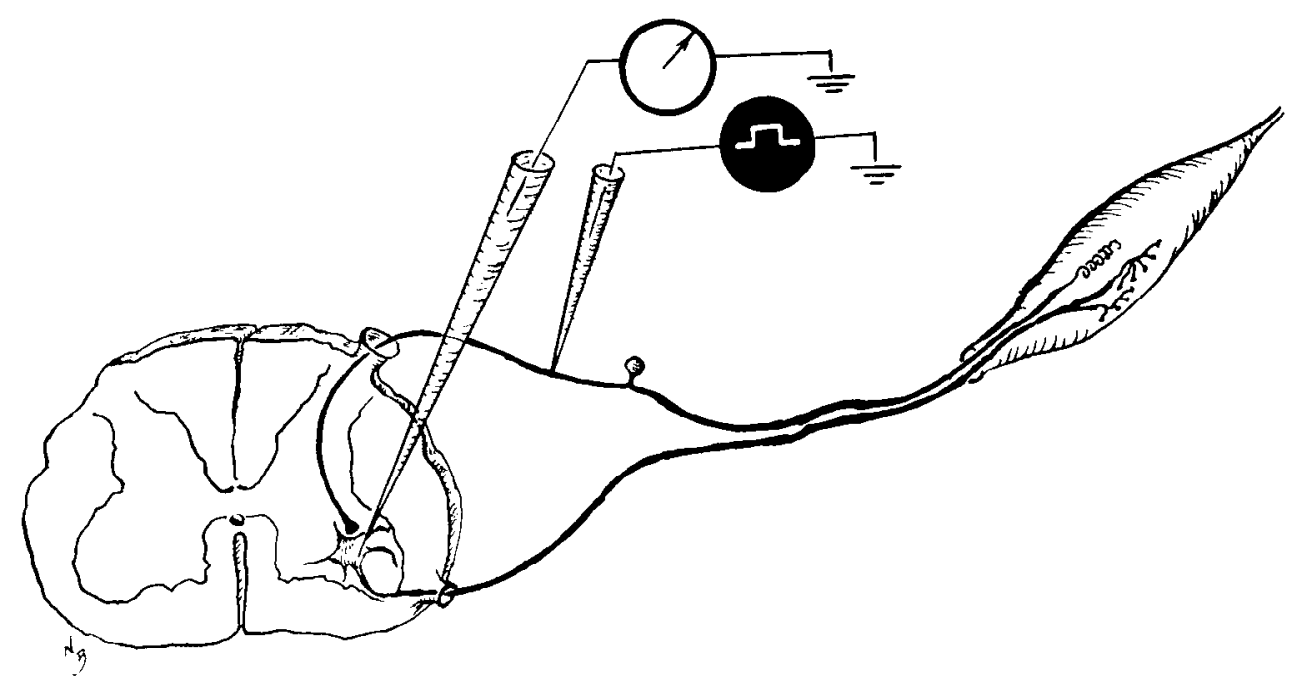

Figure 1. Diagram of the preparation illustrating the approximate positioning of the microelectrodes used for simultaneous intracellular penetrations of medial gastrocnemius motoneuron and Ia afferent fiber.

enlargement was exposed by laminectomy and an $\mathrm{S} 1$ dorsal root filament exhibiting stretch-evoked activity from the medial gastrocnemius (MG) muscle was isolated in continuity on recording hooks (Fig. 1). An MG motoneuron identified by antidromic activation of the dissected MG muscle nerve was impaled using $3 \mathrm{M} \mathrm{KCl}$ micropipettes. A second $3 \mathrm{M}$ $\mathrm{KCl}$ electrode was then lowered into the dorsal root until an $\mathrm{MG}$ group Ia fiber was impaled. In most cases, stimulation of such a group Ia fiber resulted in an EPSP in the impaled motoneuron (see Henneman and Mendell, 1981, for a review of the extent of the group Ia fiber projection to homonymous motoneurons). At each connection we first measured the amplitude of the EPSP produced by low-frequency $(18 \mathrm{~Hz})$ stimulation of the Ia fiber and the rheobase of the motoneuron (see Collins, et al., 1984b).

Fach connection was then subjected to a pattern of frequency-modulated stimulation. This pattern, provided by Dr. G. E. Loeb (Laboratory of Neural Control, NIH), was derived from the discharge of a vastus medialis primary spindle afferent recorded from an intact cat while it walked on a treadmill. It was chosen to be a representative of spindle discharge from extensor muscles during walking. It consisted of a 550 msec train of 52 impulses with intervals ranging from about 5 to $75 \mathrm{msec}$ (Fig. 2). These impulses were delivered to the afferent fiber using a microcomputer (Aim 65, Rockwell International). The pattern was repeated 64-256 times with an interval of $800 \mathrm{msec}$ between the end of one train and the beginning of the next to match the stepping rate of the animal on the treadmill. The EPSPs were averaged in register (1st, 2 nd, . . , 52nd) to allow accurate determination of the mean amplitude of each EPSP.

\section{Results}

The same pattern of stimulation was presented at 24 Ia-motoneuron connections. The response averaged in register at one of these connections is shown in Figure 2, with the timing of the stimuli given below. The mean EPSP amplitude was not constant during this train of stimulation, and the modulation of EPSP amplitude during the frequency-modulated train varied at different connections (Fig. 3-see below). In some cases (not illustrated), there was considerable DC shift in the membrane potential during the burst. This occurred primarily at connections with EPSPs exhibiting large half-widths (Mendell and Collins, 1983). We demonstrated previously that changes in EPSP amplitude are not caused by shifts in membrane potential (see discussion in Honig et al., 1983). In the present sample we detected no correlation between EPSP half-width and the extent of modulation of EPSP amplitude.

Figure 3 presents examples of amplitude modulation at two connections. On the left, the mean EPSP amplitude evoked by each stimulus is displayed as a function of time during the train. Note that the amplitude could fall during the high-frequency portion of the train (Fig. $3 A$ ). The extent to which it fell was highly variable. At other connections (e.g., Fig. 3C), EPSP amplitude was virtually unchanged during the frequency-modulated train. To the right of each of these graphs, the EPSP amplitudes generated at these connections are displayed as a function of the instantaneous frequency immediately preceding each stimulus (Fig. 3, $B$ and $D$ ). This demonstrates even more graphically the extent to which individual Ia fiber/alpha-motoneuron connections differ in the way they process these identical trains of frequency-modulated discharge patterns. At some connections there was a dramatic decrease in EPSP amplitude as frequency increased (Fig. 3, $A$ and $B$ ), whereas at others little or no difference in EPSP amplitude was observed (Fig. 3, $C$ and $D$ ).

It should not be construed from records such as Figure $3 B$ that modulation of EPSP amplitude depended solely on instantaneous frequency. The response could also depend on the cumulative effects of the multiple stimuli, as indicated in previous experimental work with bursts of trains of a single frequency (Collins et al., 1984b; Honig et al., 1983). In those experiments, we observed progressive changes in EPSP amplitude during such repetitive stimulation. This question was examined in the present work by averaging the EPSPs produced by 16 stimuli during the high-frequency portion of the frequency-modulated burst (stimuli 17 to 32) under two separate conditions: (1) embedded in the burst of 52 stimuli and (2) in isolation, i.e., in the absence of the other 36 stimuli. Thus, in the "embedded" case the response to the 16 shocks could be determined both by instantaneous frequency and by the history produced by the preceding stimuli. In the "isolated" case, the preceding (and subsequent) stimuli were absent. Thus, although the interstimulus intervals were the same as in the "embedded" case, there was a clear difference in the preceding history.

Two general patterns were observed. In one (Fig. 4, $C$ and $D)$, EPSPs in the isolated case were considerably larger than those in the embedded case in response to each stimulus in the series. In the second (Fig. 4, $A$ and $B$ ), EPSP amplitudes in the isolated case were approximately equal to those in the embedded case. These plots are shown mainly to emphasize the point that the relationship between EPSP amplitude and instantaneous stimulus frequency could be altered to a significant extent by the preceding history of stimulation. At none of the five connections studied in this way was the sign of the slope of the amplitude-frequency relationship affected by changing the history. The technical limitations inherent in these experiments requiring simultaneous penetrations of afferent fiber and alphamotoneuron precluded a more extensive study of this phenomenon. 


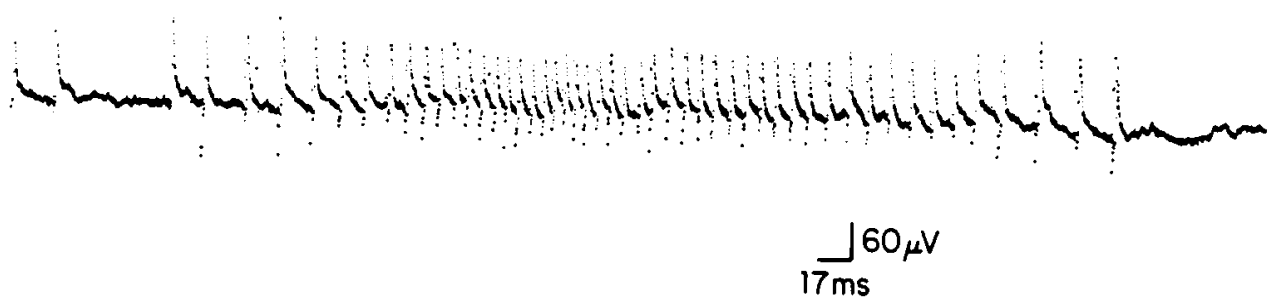

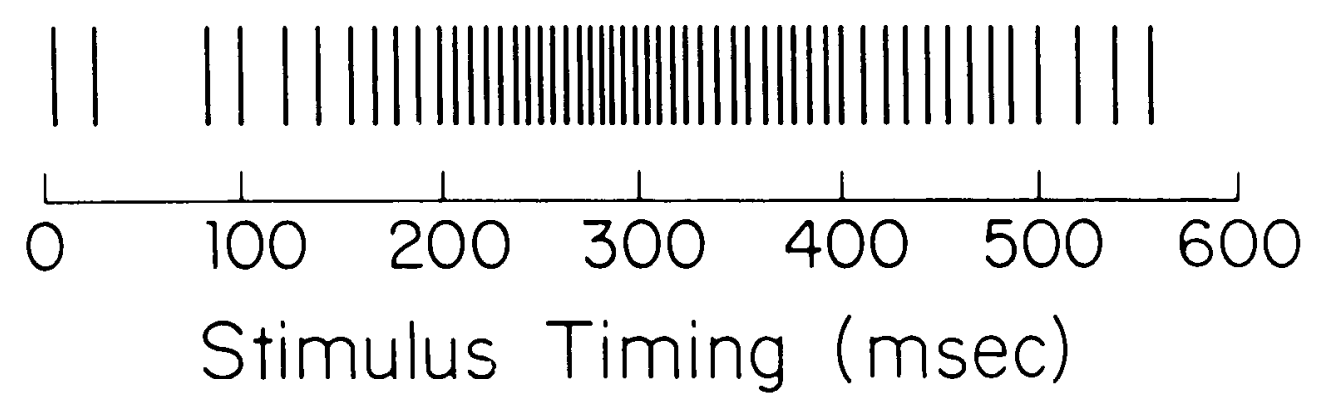

Figure 2. Example of averaged (128 trials) EPSPs recorded from MG motoneuron during frequency-modulated bursts of stimulation delivered to the impaled Ia fiber. The timing of the frequency-modulated stimuli is indicated below. Each timing (stimulus) bar in the bottom row is associated with an EPSP in the top trace.
A more quantitative approach to the amplitude modulation was undertaken, the results of which are displayed in Figure 5. We compared the amplitude of the EPSP generated at the beginning of the train, during the high-frequency portion of the middle of the train, and at the end of the train, to the amplitude of the control EPSP avcraged at a different time using $18 \mathrm{~Hz}$ stimulation. We found that the initial EPSP (which in fact occurs $800 \mathrm{msec}$ after the end of the previous train) was generally (20/ 24) larger than the amplitude obtained during low-frequency $(18 \mathrm{~Hz})$ stimulation (Fig. $5 A)$. At the highest frequency portion of the train we observed that EPSP amplitude (mean of EPSPs $23-27, \bar{\mu}_{23-27}$; ISI $\left.5.2,5.5,5.3,5.4,6.3 \mathrm{msec}\right)$ had usually (21/ 24) fallen below the value obtained during regular, low-frequency $(18 \mathrm{~Hz}$ ) stimulation (Fig. $5 B$ ). Near the end of the train (mean of EPSPs $45-49, \bar{\mu}_{45-49}$; ISI $10.7,10.6,10.6,10.6$, and $10.6 \mathrm{msec}$ ) EPSP amplitude had recovered close to the value observed during low-frequency $(18 \mathrm{~Hz})$ stimulation, although it was still slightly depressed on average (Fig. $5 \mathrm{C}$ ).

These results indicate that EPSP amplitude could increase or decrease during different portions of the frequency-modulated train of stimulation. Figure 5 suggests that connections with large EPSPs exhibited the greatest absolute increase in EPSP amplitude at the beginning of the train $\left(E P S P_{1}\right)$ and the greatest absolute decline in amplitude during the high-frequency portion of the train $\left(\bar{\mu}_{23-27}\right)$. However, we found no relationship between fractional increase or decrease in amplitude (i.e., EPSP 1 /control; $\bar{\mu}_{23-27} /$ control) as a function of control amplitude (not illustrated). We examined this question more closely by computing the following ratios: $\operatorname{EPSP}_{1} / \bar{\mu}_{45-49}$, which is an estimate of potentiation in EPSP amplitude at the beginning of the train, and $\bar{\mu}_{23-27} / \bar{\mu}_{45-49}$, which is an estimate of the depression in amplitude during the middle (high-frequency) portion of the train. The advantage of using $\bar{\mu}_{45-49}$ instead of the amplitude at $18 \mathrm{~Hz}$ (as in Fig. 5) is that it is an internal control (i.e., obtained during frequency-modulated stimulation), and therefore these ratios represent directly the extent of modulation in either direction during the frequency-modulated train. We found (Fig. 6) that connections with the greatest degree of potentiation after the burst (ordinate) were, on average, those that depressed to the greatest extent during the burst (abcissa) (Spearman rank correlation, $r=-0.42, p<0.05$ ). In other words, at some connections there was little modulation of EPSP amplitude (righthand side of Fig. 6), whereas at others the modulation was substantial, with the initial EPSP being potentiated and the EPSPs evoked at high frequency being depressed (left-hand side of Fig. 6). The results obtained using the amplitude during 18 $\mathrm{Hz}$ stimulation instead of $\bar{\mu}_{45-49}$ as a control were similar but did not reach statistical significance.

Wc investigated whether the total extent of modulation differed systematically at different connections (Fig. 7). We calculated the modulation as the ratio of the first EPSP in the burst, generally the largest one, to $\bar{\mu}_{23-27}$, representing the response during the highest frequency of stimulation. We found that EPSPs generated in cells with high rheobase exhibited littlc modulation. No cell with rheobase $>14 \mathrm{nA}$ showed modulation $>1.5$. Cells with rheobase $<8 \mathrm{nA}$ exhibited modulation $>1.3$ and often $>1.5$. Modulation was found to be correlated significantly with
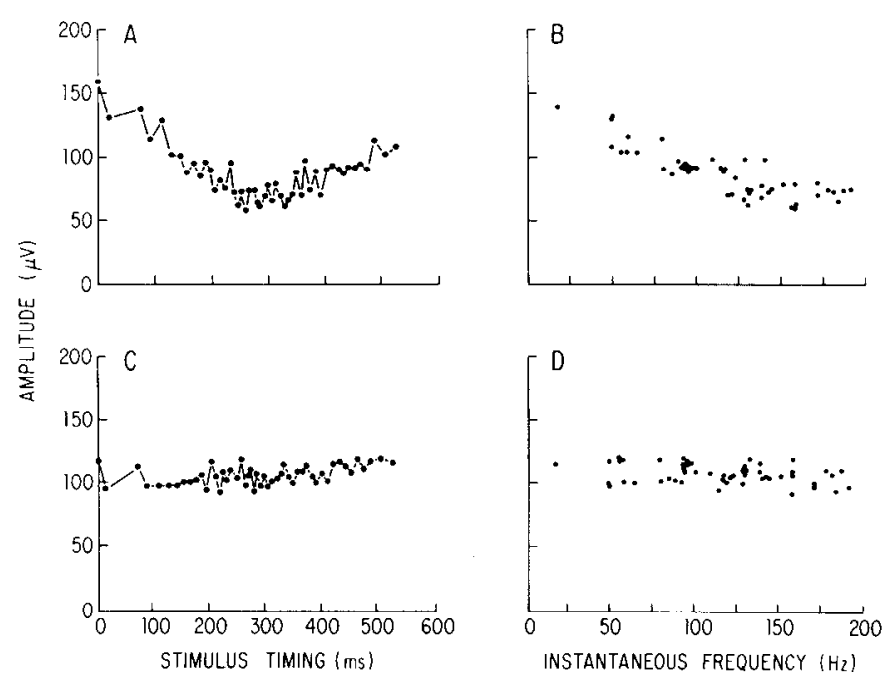

Figure 3. EPSP amplitude modulation during frequency-modulated stimulus trains. The results at two connections in different preparations are illustrated, one in $A$ and $B$ and the other in $C$ and $D . A$ and $C$, EPSP amplitude plotted as a function of time from the first stimulus in the burst. $B$ and $D$, Same data plotted as a function of instantaneous frequency (defined as reciprocal of previous interval). The graphs in $B$ and $D$ suggest that the modulation in EPSP amplitude at a given connection can be strongly $(B)$ or weakly $(D)$ related to the frequency of stimulation. 
Figure 4. Effect of prior history on EPSP amplitude during frequencywere evoked under two different conditions: (1) the shocks evoking EPSPs 17-32 were embedded within the 52 shock frequency-modulated train (i.e., normal stimulation; closed circles), or (2) stimuli evoking EPSPs 17-32 were presented alone or "isolated" without any other stimuli; open circles; see text) Data from two connections are illustrated, one in $A$ and $B$ and the other in $C$ and $D$. EPSP amplitude is plotted as a function of stimulus timing $(A, C)$ and instantaneous frequency $(C, D)$. Note for the connection illustraled in $B$ and $D$ that EPSP amplitudes were much more a function of history than instantaneous frequency, whereas at the other connection $(A, C)$ history played a much less prominent role than frequency. modulated stimulation. EPSPs 17-32
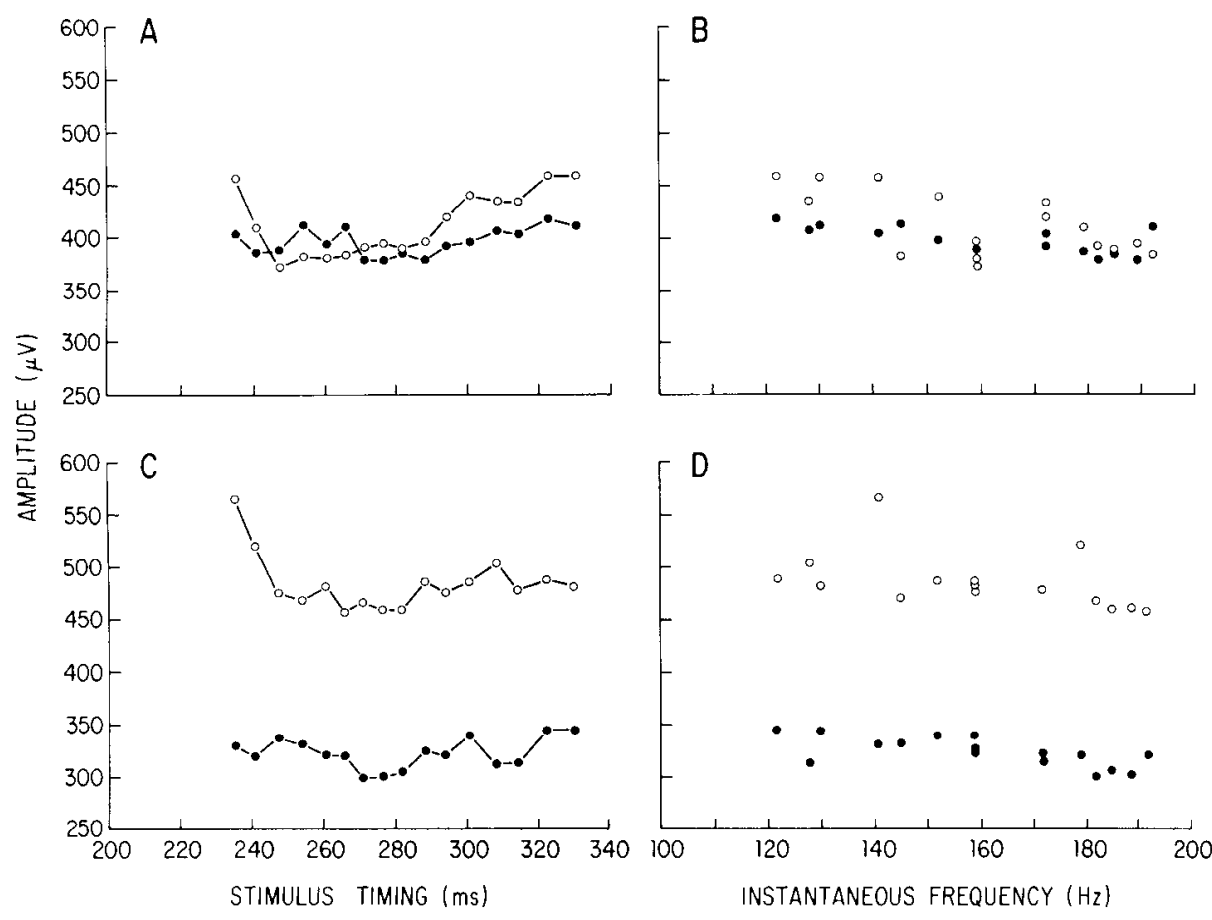

rheobase (Spearman rank correlation, $r=-0.44, p<0.05$ ) but not with EPSP amplitude. Thus, the degree of modulation under this stimulation condition seems to be partially dependent on the properties of the motoneuron on which the synaptic connection is made, presumably through its influence (e.g., trophic) on presynaptic terminals), but not on the strength of the connection itself. Separate evaluation of potentiation $\left(\operatorname{EPSP}_{1} / \bar{\mu}_{45-49}\right.$ : ordinate of Fig. 6$)$ and depression $\left(\bar{\mu}_{23-27} / \bar{\mu}_{45-49}:\right.$ abscissa of Fig. $6)$ as a function of rheobase revealed a negative correlation between $\operatorname{EPSP}_{\mathrm{i}} / \bar{\mu}_{45-49}$ and rheobase (Spearman $r=0.49, p<$ $0.02)$ and a positive correlation between $\bar{\mu}_{23-27} / \bar{\mu}_{45-49}$ and rheobase (Spearman $r=0.35, p<0.15$ ), but as discussed above in conjunction with Figure 5, no correlation with amplitude was seen (Spearman $|r|<0.2, p>0.1$ ).

\section{Discussion}

The present studies demonstrate the importance of using naturally occurring patterns of discharge to analyze synaptic function. Previous work has been carried out using single frequencies of stimulation. Nlthough such studies indicated that EPSP amplitude is a function of stimulation frequency (Collins et al., 1984b; Curtis and Eccles, 1960; Honig et al., 1983; Luscher et al., 1981), the physiological role of such modulation has always been uncertain. One key advance allowing the present studies to be carried out is the technically demanding procedure of recording impulse patterns in fibers innervating identified muscle receptors in walking cats (Loeb and Duysens, 1979; Prochazka et al., 1976). We delivered one such pattern repetitively to single afferent fibers and found that amplitude modulation of EPSPs occurs when natural patterns of Ia fiber activation (i.e., frequency modulated) are used.

The amplitude modulation we have observed is complex and involves several processes. One process seems to cause reduction of EPSP amplitude during high-frequency stimulation, whereas another results in potentiation of EPSP amplitude following the train (as indicated by the increased amplitude of EPSP $_{1}$ ). Furthermore, there appears to be somecorrelation among these processes, such that connections exhibiting the most potentiation are those that depress the most during the train. This pattern of stimulation seems to evoke a much greater degree of amplitude modulation of EPSPs at some connections than at others. The connections at which this modulation are greatest are on low-rheobase motoneurons.

The functional significance of the complcx pattcrn of amplitude modulation can only be speculated on at present. It may mean that at the onset of the frequency-modulated train, the greatest synaptic drive is provided to low-rheobase motoneurons supplying type $\mathbf{S}$ motor units (Fleshman et al., 1981a) because of both the larger EPSPs in such cells (Fleshman et al., $1981 \mathrm{~b}$ ) and the greater level of potentiation of these EPSPs (see below). Thus, the recruitment of these motoneurons would be favored, as previously shown for the motoneurons of slowest conduction velocity (see review in Henneman and Mendell, 1981) or smallest twitch tension (Milner-Brown et al., 1973; Zajac and Faden, 1985), both of which are putatively type $S$ units. As the frequency of afferent drive increases, EPSP amplitude decreases primarily in low-rheobase motoneurons. We do not know whether this effect is sufficiently large to reverse recruitment order, i.e., cause type $S$ motoneurons to stop firing, leaving type $\mathrm{F}$ motoneurons still active. A more conservative hypothesis derives from the supposition that temporal summation during high-frequency activation would cause type $\mathrm{S}$ motoneurons, which on average generate larger EPSPs and have longer time constants (Fleshman et al., $1981 \mathrm{a}, \mathrm{b}$ ), to depolarize to a much greater extent than type F motoneurons, which develop smaller EPSPs. This depolarization of type $\mathrm{S}$ motoneurons could be excessive in the sense that it might render the motoneuron insensitive to postsynaptic inhibitory influences. The differences in EPSP modulation might be viewed, then, as a mechanism whereby the Ia fiber, whose inputs are distributed to virtually all motoneurons in the homonymous pool (Fleshman et al., 1981b; Mendell and Henneman, 1971), could provide the appropriate intensity of synaptic drive to these diverse motoneurons over a wide range of frequency of afferent discharge. In other words, type $S$ motoneurons would be favored at the onset, i.e., be recruited first, but would not be driven excessively during the high-frequency portion of the afferent discharge. Such speculations concerning the applicability of these 

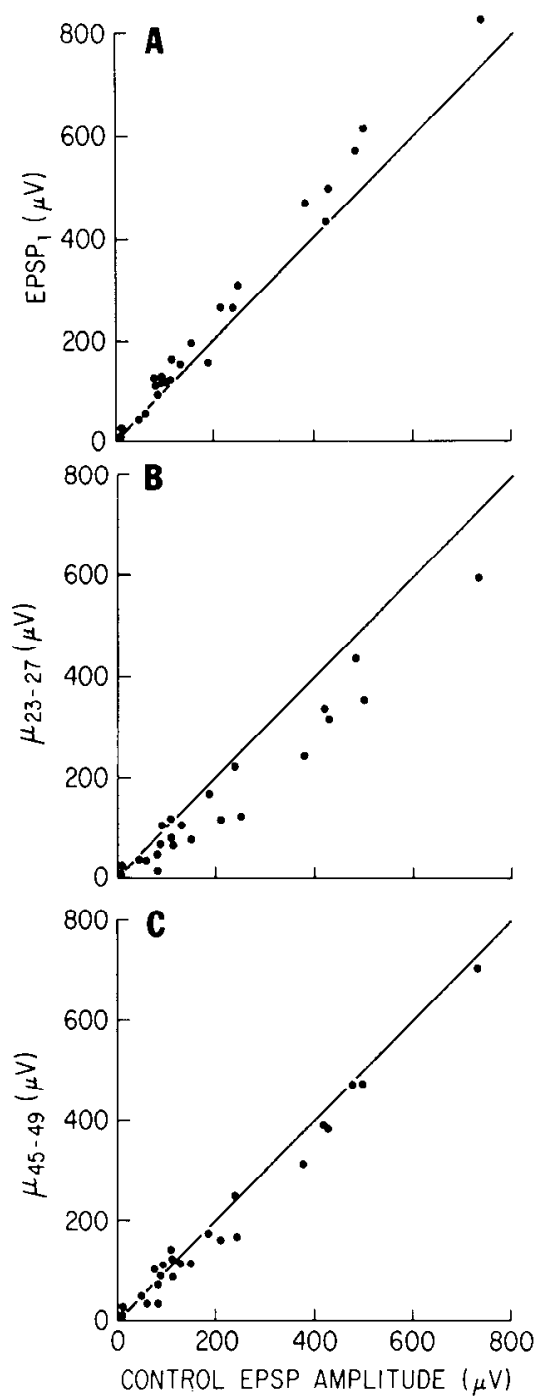

Figure 5. A, Graph of the amplitude of the averaged first EPSP in the burst (EPSP ${ }_{1}$ ) versus the amplitude of the averaged EPSP during control $(18 \mathrm{~Hz})$ stimulation. Note that the first EPSP in the burst is usually larger in amplitude than the EPSP during control stimulation (potentiation). $B$, Graph of average amplitude of five EPSPs (23-27) during the high-frequency portion (average instantaneous frequency $=174 \mathrm{~Hz}$ ) of the burst plotted as a function of the amplitude of the EPSP averaged during control stimulation. In most cases, EPSP amplitude during the high-frequency portion of the burst is less than during the $18 \mathrm{~Hz}$ stimulation (depression). $C$, Graph of the average amplitude of tive EPSP (45-49) evoked during the low-frequency (average instantaneous frequency $=92 \mathrm{~Hz}$ ) portion of the end of the burst. These values were very similar to those seen using control stimulation. The line in the three graphs indicates equality of EPSPs during the burst (i.e., EPSP ${ }_{1}$, $\left.\bar{\mu}_{23-27}, \bar{\mu}_{43-49}\right)$ to the control EPSP.

findings to normal function depend on the assumption that the differences we have observed are not a consequence of the deep barbiturate anesthesia used here.

Our previous data indicate that high-frequency activation of group Ia fibers converging on the same motoneuron tends to elicit similar patterns of amplitude modulation (Honig et al., 1983). Thus, the differences in EPSP amplitude modulation described in the present work, which are modest at the level of single connections, would tend to be magnified by the convergence of 50-60 group Ia afferents onto each motoneuron.

The processes that appear to be operating here are qualitatively similar to those we have observed previously using short

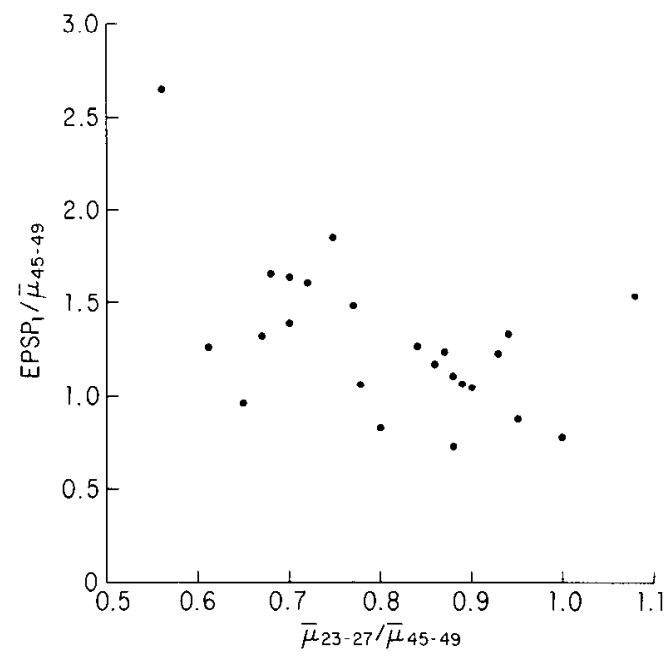

Figure 6. Relationship between EPSP potentiation and depression during frequency-modulated trains of stimulation. Potentiation is represented by the ratio of EPSP, (generally the largest EPSP in the burst) divided by the average amplitude of EPSPs $45-49\left(\bar{\mu}_{45-49}\right)$. The average amplitude of F.PSP 45-49 was used because this value was very close to that of the control (i.e., $18 \mathrm{~Hz}$ ) EPSP (Fig. 5C) and because it provided an internal control (i.e., it was obtained from EPSPs occurring during the burst). Depression is represented by the average of EPSPs 23-27 $\left(\bar{\mu}_{23-27}\right)$, the smallest EPSPs in the burst, divided by $\bar{\mu}_{45-49}$. The graph shows that the connections that potentiated the most also exhibited the most depression (Spearman rank correlation $r=-0.42, p<0.05$ ).

(32 shocks), high-frequency bursts of a single frequency (167 $\mathrm{Hz}$ ). During such bursts one can observe either a decrease (depression) or an increase (facilitation) in EPSP amplitude (Collins et al., 1984a; Honig et al., 1983). It seems likely that both processes actually occur simultaneously, with the observed change being the net result (Collins et al., 1984b). Under the present conditions of stimulation we have noted a greater net depression, but this may be a consequence of the different mode of stimulation, e.g., the gradual increase in frequency of stimulation rather than the abrupt increase in stimulation frequency that necessarily occurs when bursts of single high frcquency arc

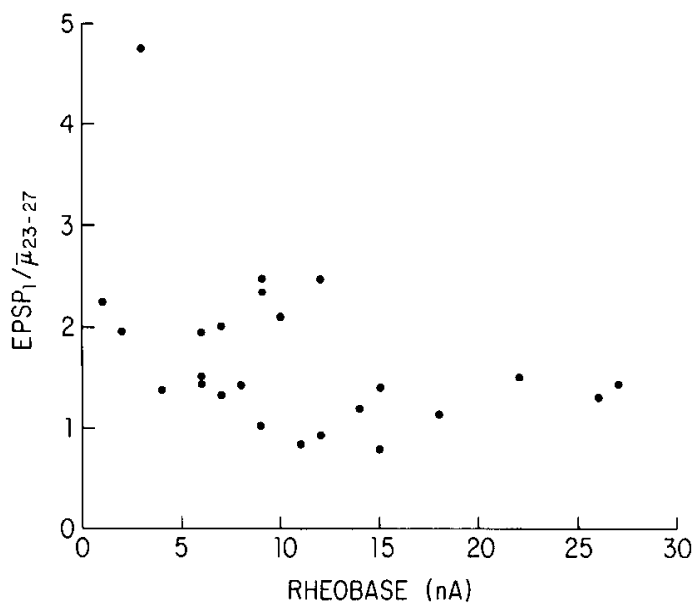

Figure 7. Extent of EPSP amplitude modulation as a function of motoneuron rheobase. Modulation of EPSP amplitude during the stimulation train is represented by the ratio of EPSP, to the average of five EPSPs occurring during the high-frequency portion of the stimulus train $\left(\bar{\mu}_{23-27}\right)$. The greatest amount of modulation was seen at connections on small rheobase motoneurons. Modulation was significantly correlated with motoneuron rheobase. (Spearman rank correlation $r=-0.44, p<$ 0.05.) 
used. These differences in stimulation parameters may also account for the puzzling finding that these variables were related to motoneuron rheobase but not EPSP amplitude, in contrast to our previous results (Collins et al., 1984b). The potentiation observed in the present experiments is qualitatively similar to that observed after single, high-frequency $(167 \mathrm{~Hz}$ ) bursts (Davis et al., 1986) but is of smaller magnitude, despite instantaneous frequencies approaching $200 \mathrm{~Hz}$; presumably this occurred because the mean frequency of stimulation $(93 \mathrm{~Hz})$ was lower. Our results indicating that this process is observed after impulse trains similar to those occurring under physiological conditions suggest that potentiation is important in determining synaptic strength during normal motor behavior.

Other workers have reported that posttetanic potentiation is greatest on low-resistance motoneurons (Lev Tov et al., 1983; Luscher et al., 1983), which tend to have high values of rheobase (Fleshman et al., 1981a). This is in apparent contradiction to our finding that potentiation, defined as $\operatorname{EPSP}_{i} / \mu$, was negatively correlated with motoneuron rheobase, i.e., connections with high values of potentiation were on motoneurons with low values of rheobase. These differences in the distribution of potentiation can be explained by the results of experiments in which single Ia fibers were repeatedly stimulated with short, high-frequency bursts, followed by a single test shock during the 2 sec interburst interval (Davis et al., 1985). It was found that the maximum value of potentiation was achieved within $100 \mathrm{msec}$ after the burst, and this maximum potentiation was greatest in highrheobase motoneurons (as expected from the work of Luscher et al., 1983, and I ev Tov et al., 1983). However, potentiation decayed more quickly in high-rheobase motoneurons, such that at $2 \mathrm{sec}$ after the burst, potentiation was greatest in small rheobase motoneurons, i.e., the relative magnitude of potentiation in different motoneurons reverses in the period from 50-100 msec to 2 sec. Thus, in the present experiments, we presume that the distribution of potentiation in motoneurons $800 \mathrm{msec}$ after the burst approaches more closely that seen $2 \mathrm{sec}$ after the burst than 50-100 msec after the burst. Under these conditions, the order of recruitment at train onset established by the distribution of EPSP amplitudes would be accentuated by the distribution of potentiation, rather than being reversed (see Zajac and Faden, 1985, p. 1318).

These results support the contention that EPSP amplitude is not fixed at a given connection. This conclusion had been reached previously using high-frequency bursts (Collins et al., 1984b; Davis et al., 1985), but the applicability of this finding to physiological function could not be established with certainty. The ability to analyze synaptic function using more natural stimulation parameters has revealed that these modulatory mechanisms operate even in response to realistic frequency-modulated inputs. However, they are not the same at all connections, since some experience a much greater degree of EPSP amplitude modulation than others. Taken together, these findings imply that the amplitude of an EPSP established during low-frequency stimulation cannot be used to establish the "strength" or "efficacy" of a particular connection that applies under all conditions. From a more functional point of view, one can infer that different connections made by the same afferent on different motoneurons can exhibit very different transmission properties, although this remains to be proven directly by studying the projection from one afferent to many different motoneurons.

\section{References}

Burke, R. E. (1981) Motor units: Anatomy, physiology, and functional organization. In Handbook of Physiology, Vol. 2: The Nervous Sys- tem, Sec. 1, Part 1, chap. 10, pp. 345-421, American Physiological Society, Bethesda, MD.

Collins, W. F., III, B. M. Davis, and L. M. Mendell (1984a) Variation of spinal motoneurons EPSP amplitude during frequency modulated stimulation of single Ia afferent fibers. Soc. Neurosci. Abstr. 10:742.

Collins, W. F., III, M. G. Honig, and L. M. Mendell (1984b) Heterogeneity of group Ia synapses on homonymous alpha-motoneurons as revealed by high frequency stimulation of Ia afferent fibers. J. Neurophysiol. 52: 980-993.

Curtis, D. R., and J. C. Eccles (1960) Synaptic action during and after repetitive stimulation. J. Physiol. (Lond.) 150: 374-398.

Davis, B. M., W. F. Collins, III, and L. M. Mendell (1985) Potentiation of transmission of Ia-motoneuron connections induced by repeated short bursts of afferent activity. J. Neurophysiol. 54: 1541-1552.

Evarts, E. V. (1981) Role of motor cortex in voluntary movements in primates. In Handbook of Physiology, Vol. 2: The Nervous System, Sec. 1, Part 1, chap. 23, pp. 1083-1120, American Physiological Society, Bethesda, MD.

Fleshman, J. W., J. B. Munson, G. W. Sypert, and W. A. Friedman (1981a) Rheobase, input resistance and motor unit type in medial gastrocnemius motoneurons of the cat. J. Neurophysiol. 46: 13261338 .

Fleshman, J. W., J. B. Munson, and G. W. Sypert (1981b) Homonymous projection of individual group Ia-fibers to physiologically characterized medial gastrocnemius motoneurons in the cat. J. Neurophysiol. 46: 1339-1348.

Henneman, E., and L. M. Mendell (1981) Functional organization of motoneuron pool and its inputs. In Handbook of Physiology, Vol. 2: The Nervous System. Sec. 1, Part 1, chap. 11, pp. 423-507, American Physiological Society, Bethesda, MD.

Honig, M. G., W. F. Collins, III, and L. M. Mendell (1983) $\alpha$-Motoneuron EPSPs exhibit different frequency sensitivities to single Iaafferent fiber stimulation. J. Neurophysiol. 49: 886-901.

Lev Tov, A., M. J. Pinter, and R. E. Burke (1983) Posttetanic potentiation of group Ia fibers: Possible mechanisms for differential distribution among medial gastrocnemius motoneurons. J. Neurophysiol. 50: $379-398$

Loeb, G., and J. Duysens (1979) Activity patterns in individual hindlimb primary and secondary muscle spindle afferents during normal movements in unrestrained cats. J. Neurophysiol. 42: 420-440.

Luscher, H.-R., P. Ruenzel, and E. Henneman (1981) Factors that influence distribution of input from group Ia and group II spindle afferents to pools of motoneurons. Adv. Physiol. Sci. 16: 249-257.

Luscher, H.-K., P. Kuenzel, and E. Henneman (1983) Composite EPSPs in motoneurons of different sizes before and during PTP: Implications for transmission failure and its relief in Ia projections. J. Neurophysiol. 49: 269-289.

Mendell, L. M., and W. F. Collins, III (1983) Determination of synaptic efficacy at individual Ia-motoneuron connections. Soc. Neurosci. Abstr. 9: 664 .

Mendell, L. M., and E. Henneman (1971) Terminals of single Ia fibers: Location, density and distribution within a pool of 300 homonymous motoneurons. J. Neurophysiol. 34: 171-187.

Milner-Brown, H. S., R. B.. Stein, and R. G. Yemm (1973) The orderly recruitment of human motor units during voluntary isometric contractions. J. Physiol. (Lond.) 230: 359-370.

Prochazka, A., R. A. Westerman, and S. P. Zicone (1976) Discharges of single hindlimb afferents in the freely moving cat. J. Neurophysiol. 39: 1090-1104.

Prochazka, A., R. A. Westerman, and S. P. Zicone (1977) Ia afferent activity during a variety of voluntary movements in the cat. J. Physiol. (Lond.) 268: 423-448.

Somjen, G. G. (1972) Sensory Coding in the Mammalian Nervous System, Appleton-Century-Crofts, New York.

Zajac, F. E., and J. S. Faden (1985) Relationship among recruitment order, axonal conduction velocity, and muscle-unit properties of typeidentified motor units in cat plantaris muscle. J. Neurophysiol. 53: 1303-1322.

Zengel, J. E., Munson, J. B. and Sypert, G. W. (1985) Membrane electrical properties of type-identified medial gastrocnemius motoneurons in the cat. J. Neurophysiol. 53: 1323-1344. 